\title{
High-Order Circular Derivative Pattern for Image Representation and Recognition
}

\author{
Sanqiang Zhao \\ QRL, National ICT Australia \\ IIIS, Griffith University \\ Brisbane, Queensland, Australia \\ sam.zhao@nicta.com.au \\ s.zhao@griffith.edu.au
}

\author{
Yongsheng Gao \\ School of Engineering, Griffith University \\ QRL, National ICT Australia \\ Brisbane, Queensland, Australia \\ yongsheng.gao@griffith.edu.au \\ yongsheng.gao@nicta.com.au
}

\author{
Terry Caelli \\ Queensland Research Laboratory \\ National ICT Australia (NICTA) \\ Level 5, Axon Building, St Lucia \\ Brisbane, Queensland, Australia \\ terry.caelli@nicta.com.au
}

\begin{abstract}
Micropattern based image representation and recognition, e.g. Local Binary Pattern (LBP), has been proved successful over the past few years due to its advantages of illumination tolerance and computational efficiency. However, LBP only encodes the first-order radial-directional derivatives of spatial images and is inadequate to completely describe the discriminative features for classification. This paper proposes a new Circular Derivative Pattern (CDP) which extracts highorder derivative information of images along circular directions. We argue that the high-order circular derivatives contain more detailed and more discriminative information than the first-order LBP in terms of recognition accuracy. Experimental evaluation through face recognition on the FERET database and insect classification on the NICTA Biosecurity Dataset demonstrated the effectiveness of the proposed method.
\end{abstract}

Keywords-Image representation; image recognition; micropattern representation; Circular Derivative Pattern

\section{INTRODUCTION}

Micropattern based image representation and recognition, e.g. Local Binary Pattern (LBP), has received noticeable attention over the past few years. The original LBP operator, proposed by Ojala et al. [1, 2], was first used for texture analysis, but later was extended to other research areas such as face detection [3], face recognition [4], expression analysis [5], and background modeling [6]. The LBP representation has the advantages of high discrimination capacity, invariance against monotonic gray-scale changes and computational efficiency. There have been a lot of research efforts devoted to improving the performance of LBP. For example, Zhao et al. [7] proposed a Sobel-LBP to enhance the performance of LBP using the Sobel operator. Zhang et al. [8] proposed a Local Derivative Pattern (LDP) to encode high-order multi-directional micropatterns for face recognition. Both methods obtained better recognition performances than LBP.

Different from the abovementioned LBP variants, this paper proposes a new high-order Circular Derivative Pattern (CDP) for image representation and recognition. The CDP operator extracts the high-order derivative features along the circular direction of an image pixel at the micropattern level. It is different from the LBP operator that encodes the firstorder derivative features along the radial directions. We argue that the high-order derivative features contain more detailed and more discriminative information that can be employed for better classification. Because the high-order derivatives tend to be sensitive to noise when the order becomes high, we use a specially designed thresholding function to preserve the coarse gradient change information instead of conventional difference information used in the first-order LBP. The statistical spatial histogram of CDPs is used to represent an image. To evaluate the proposed method, comparative experiments of face recognition and insect classification are conducted on the publicly available FERET database [9] and the NICTA Biosecurity Dataset, respectively. The experimental results demonstrate that the high-order CDP micropatterns exhibit higher discrimination capacity than the first-order LBP. However, when the order of CDP further increases, the performance degrades as overhigher-order micropattern features contain more noise than the identify information.

The remaining part of this paper is organized as follows. Section II describes the proposed high-order CDP operator in detail. Section III reports the comparative experiments. Section IV concludes the paper with some discussions.

\section{High-ORDER CIRCUlAR DERIVATIVE PATTERN}

Mathematically, the Local Binary Pattern (LBP) marks each pixel $I_{c}$ of an image as a decimal number $L B P_{P, R}\left(I_{c}\right)$, which is formed by thresholding the $P$ equally spaced neighbor pixels $I_{p, R}(p=0, \cdots, P-1)$ on a circle of radius $R$ with the center pixel $I_{c}$ and concatenating the results binomially with factor $2^{p}$. If the coordinate of $I_{c}$ is $(0,0)$, then the coordinates of $I_{p, R}$ are computed by $(-R \sin (2 \pi p / P), R \cos (2 \pi p / P))$. The gray-level values of neighbors $I_{p, R}$ not falling exactly in the center of pixels can be estimated by interpolation.

\section{A. $\quad N^{\text {th }}$-Order Circular Derivative Pattern}

Theoretically, if a spatial image is represented in a polar coordinate $I(r, \theta)$ (see Fig. 1), then LBP can be conceptually considered as the first-order derivative micropattern features extracted along the radial direction (i.e. $\partial I / \partial r)$ in the polar coordinate. In this paper, the Circular Derivative Pattern (CDP) is proposed to extract the $n^{\text {th }}$-order 
derivative micropattern features along the circular direction (i.e. $\partial^{n} I / \partial \theta^{n}$ ).

Specifically, the first-order circular derivatives of an image is defined as

$$
I_{p, R}^{\prime}=\frac{\partial I_{p, R}(r, \theta)}{\partial \theta}=I_{p+1, R}-I_{p, R}
$$

Similarly, the second-order and the $(n-1)^{\text {th }}$-order circular derivatives are calculated, respectively, by

$$
\begin{aligned}
& I_{p, R}^{\prime \prime}=I_{p+1, R}^{\prime}-I_{p, R}^{\prime}, \\
& I_{p, R}^{(n-1)}=I_{p+1, R}^{(n-2)}-I_{p, R}^{(n-2)} .
\end{aligned}
$$

In general, the $n^{\text {th }}$-order CDP is defined as

$$
C D P_{P, R}^{(n)}=\sum_{p=0}^{P-1} t\left(I_{p+1, R}^{(n-1)}, I_{p, R}^{(n-1)}\right) \times 2^{p},
$$

where the thresholding function $t(\bullet, \bullet)$ is defined as

$$
t\left(I_{p+1, R}^{(n-1)}, I_{p, R}^{(n-1)}\right)=\left\{\begin{array}{ll}
1, & I_{p+1, R}^{(n-1)} \times I_{p, R}^{(n-1)} \geq 0 \\
0, & I_{p+1, R}^{(n-1)} \times I_{p, R}^{(n-1)}<0
\end{array} .\right.
$$

Fig. 2 illustrates the process of computing a CDP micropattern from the neighborhood of an image pixel. To get the $n^{\text {th }}$-order CDP, we first calculate the $(n-1)^{\text {th }}$-order circular derivatives, and then the thresholding function $t(\bullet, \bullet)$ encodes another-order derivative information from each of the two circularly neighboring pixels. Finally the thresholded results are concatenated binomially into a decimal number. Therefore, CDP conceptually encodes the high-order derivative information from images. The high-order derivatives contain more detailed and more discriminative information than the first-order derivatives used in LBP, and thus provide a better recognition performance.

It should be noted that CDP uses a specially designed thresholding function $t(\bullet, \bullet)$, which is different from the conventional difference thresholding function $s(\bullet, \bullet)$ used in LBP:

$$
s(x, y)=\left\{\begin{array}{ll}
1, & x-y \geq 0 \\
0, & x-y<0
\end{array} .\right.
$$

The thresholding function $t(\bullet, \bullet)$ encodes only the coarse gradient change information. It can reduce, to a certain extent, the sensitivity to noise problem, which is introduced by the high-order derivative operation of CDP. This can be explained by the example illustrated in Fig. 3, where three pixels $P 1, P 2$ and $P 3$ form an image slope. Suppose the pixel $P 2$ is contaminated with high-frequency noise and its graylevel value changes into $P 2^{\prime}$ (marked with dotted red line), $t(\bullet, \bullet)$ can obtain a same robust binary number 1 , while $s(\bullet, \bullet)$ gives different binary numbers. This example demonstrates that $t(\bullet, \bullet)$ is more robust against noise than $s(\bullet, \bullet)$.

Fig. 4 illustrates visualized examples of LBP and CDP representations for a face image from the FERET database. It is easy to observe that CDP can extract more detailed highorder information than LBP.

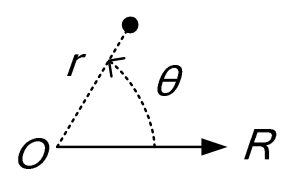

Figure 1. Spatial image in a polar coordinate.

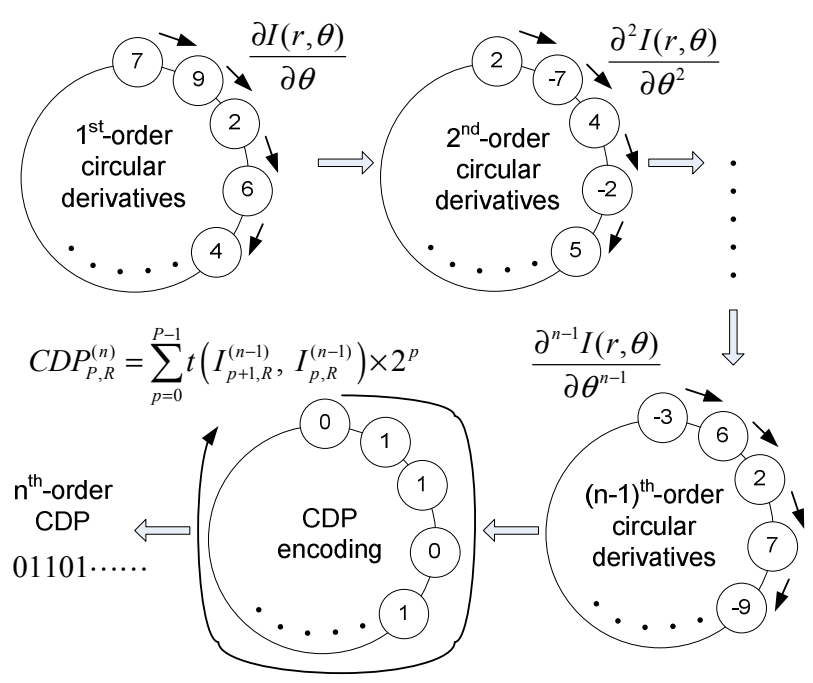

Figure 2. Computing the $n^{\text {th }}$-order Circular Derivative Pattern.

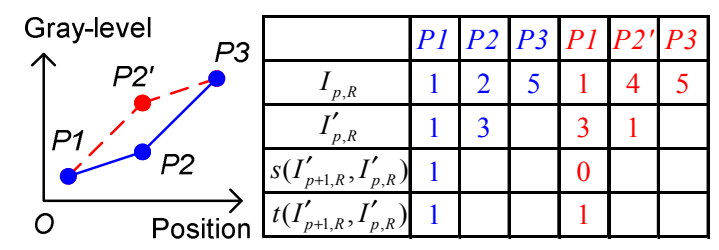

Figure 3. The robustness of $t(\cdot, \cdot)$ against noise.

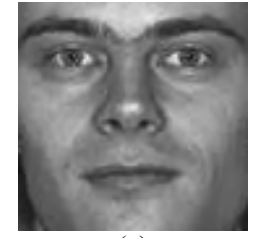

(a)

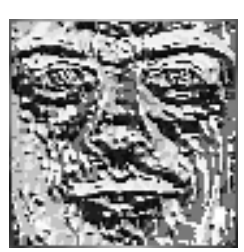

(b)

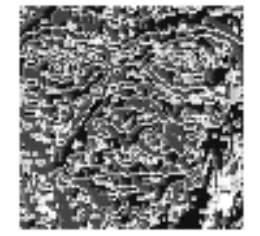

(c)
Figure 4. Visualization of LBP and CDP representations $(P=8 ; R=1)$. (a) Original image. (b) LBP. (c) Second-order CDP. 


\section{B. Spatial Histogram of CDPs}

The statistical histogram of CDP micropatterns contains important discriminative information of local features in an image. However, a holistic CDP histogram extracted from the entire image region discards the structural or shape information. To solve this problem, the spatial histogram is used to preserve the structural information of the image. Specifically, given an image spatially divided into $L$ nonoverlapping rectangular subregions, the spatial histogram of CDPs is represented as

$$
H C D P=\left\{H C D P\left(R_{k}\right) \mid k=1, \cdots, L\right\},
$$

where $R_{k}$ stands for the $k^{\text {th }}$ subregion, and $\operatorname{HCDP}\left(R_{k}\right)$ stands for the histogram of CDPs extracted from subregion $R_{k}$.

The comparison of two spatial histograms of CDPs can be conducted by any histogram measurement techniques. In this paper, we employ the histogram intersection for simplicity and efficiency:

$$
H I\left(H C D P^{1}, H C D P^{2}\right)=\sum_{j=1}^{B} \min \left(H C D P_{j}^{1}, H C D P_{j}^{2}\right),
$$

where $H^{1}$ and $H^{2}$ are two histograms, and $B$ is the number of histogram bins for both two histograms. This measurement calculates the common parts of two histograms and requires only very simple operations.

\section{EXPERIMENTAL RESULTS}

The comparative experiments between CDP and LBP are conducted on the publicly available FERET face database [9] for face recognition, and on the NICTA Biosecurity Dataset for insect classification.

\section{A. Face Recognition Experiment}

For the FERET face database, we use the frontal face images and follow the conventional experimental protocol with the gallery set (fa) and four probe sets ( $\mathrm{fb}$, fc, Dup I and Dup II). All these images are normalized and cropped into $88 \times 88$ pixels based on the positions of two eyes provided by the FERET system.

Fig. 5 illustrates the comparative rank-1 recognition rates of CDP and LBP on the four probe sets of FERET database. The subregion size of $4 \times 4$ pixels with 32 equally quantized histogram bins in each subregion is used in this experiment. With the parameter of $P=8$ and $R=1$, the average performance is increased from the first-order LBP $(62.16 \%)$ to the second-order CDP $(70.22 \%)$, and then reduced to the third-order CDP (59.34\%). With the parameter of $P=8$ and $R=2$, the same trend is observed. These results reveal that the high-order CDP can extract more discriminative information than the first-order LBP. The thresholding function in CDP can reduce the noise sensitivity problem in the high-order derivative images. However, it is incapable of dealing with further detailed information contained in the over-higherorder derivative images such as the third-order CDP.

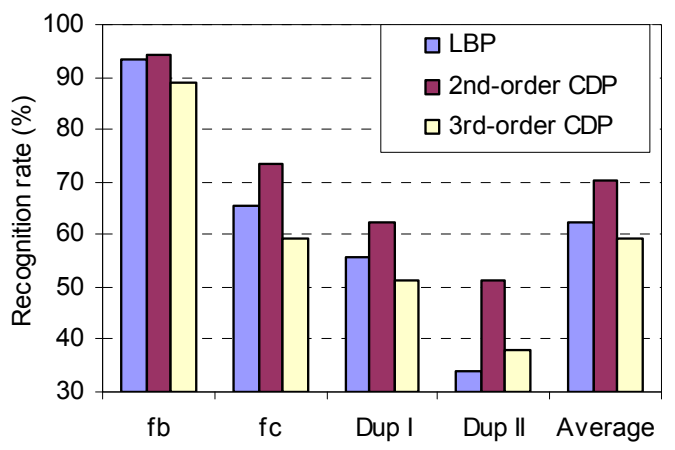

(a)

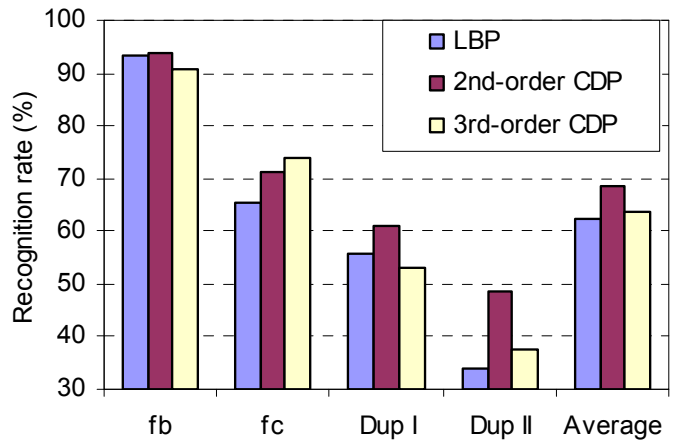

(b)

Figure 5. Rank-1 recognition accuracies of CDP and LBP on FERET database with different radiuses. (a) $P=8 ; R=1$. (b) $P=8 ; R=2$.

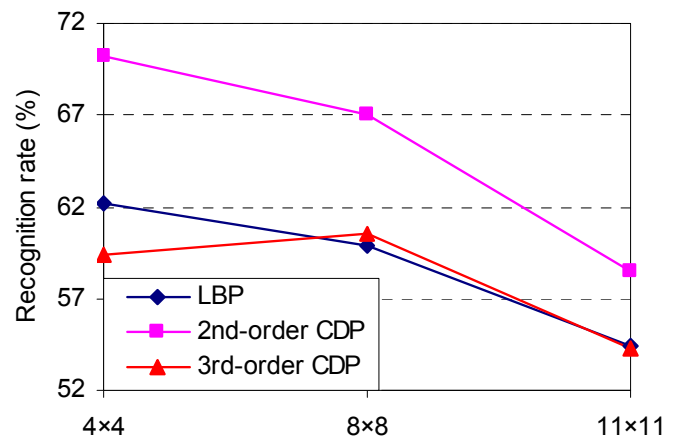

(a)

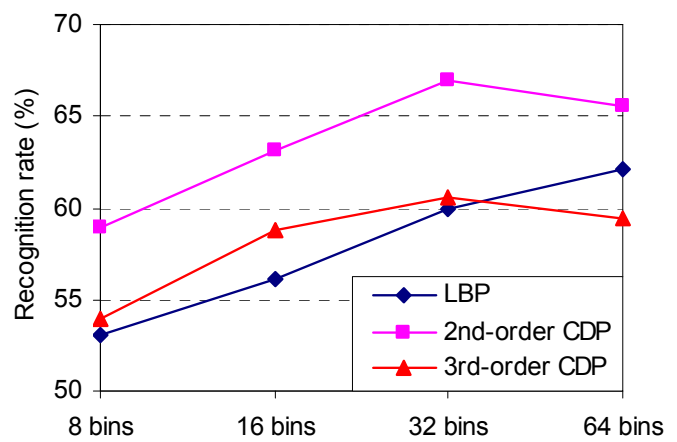

(b)

Figure 6. Rank-1 recognition accuracies of CDP and LBP on FERET database with different subregion sizes (a) and histogram bins (b). 
TABLE I. IMAGE SETS USED FOR INSECT ClASSIFICATION

\begin{tabular}{|c|c|l|}
\hline $\begin{array}{c}\text { Image } \\
\text { Set }\end{array}$ & $\begin{array}{c}\text { Image } \\
\text { Number }\end{array}$ & \multicolumn{1}{c|}{ Description } \\
\hline D1 & 60 & Regular imaging condition. \\
\hline D2 & 60 & Alternative regular imaging condition to D1. \\
\hline D3 & 57 & Different background. \\
\hline D4 & 60 & Different illumination. \\
\hline D5 & 60 & Different camera focus/scale. \\
\hline
\end{tabular}

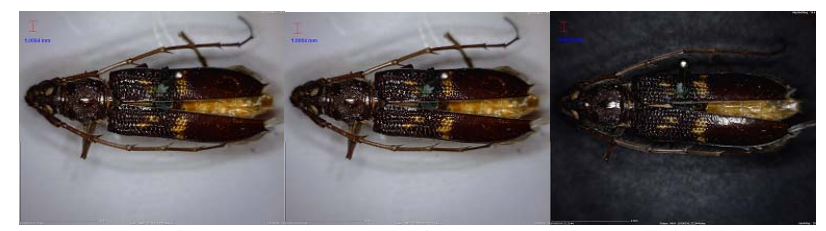

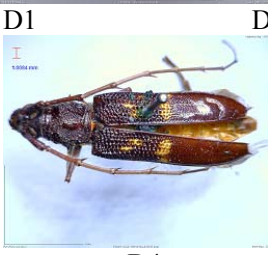

D4
D2

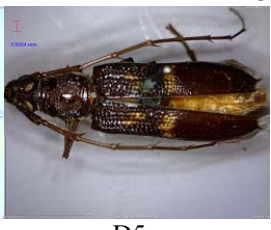

D5
Figure 7. Example images of a Phoracantha obscura sample.

TABLE II. RANK-1 CLASSIFICATION ACCURACIES OF CDP AND LBP ON NICTA BIOSECURITY DATASET

\begin{tabular}{|c|c|c|c|}
\hline & LBP & $\mathbf{2}^{\text {nd }}-$ order CDP & $\mathbf{3}^{\text {rd }}$-order CDP \\
\hline $\begin{array}{c}\text { Classification } \\
\text { Rate (\%) }\end{array}$ & 48.52 & 50.63 & 51.90 \\
\hline
\end{tabular}

To exclude the possibility that the parameters used in the previous experiments are not finely tuned in favor of LBP, we vary the subregion size and the number of histogram bins and perform the experiments with the parameter $P=8$ and $R=1$ again. The average recognition results with three different subregion sizes $(4 \times 4,8 \times 8$ and $11 \times 11)$ and the same 32 histogram bins in each subregion are reported in Fig. 6(a). The average recognition results with four different histogram bins $(8,16,32$ and 64$)$ and the same $8 \times 8$ subregion size are reported in Fig. 6(b). All these experimental results demonstrate the same trend and again prove our earlier conclusions.

\section{B. Insect Classification Experiment}

For the NICTA Biosecurity Dataset, we use 60 different beetle samples to conduct the insect classification experiment. All the beetle samples are species of Phoracantha (a genus of Cerambycidae) and native to Australia. Five images (D1, D2, D3, D4 and D5) are captured for each sample from the dorsal view under different conditions. The details are listed in Table I. Example images of a Phoracantha obscura sample are displayed in Fig. 7. In this experiment, the D1 images are used to form the gallery set, and the D2, D3, D4 and D5 images form the probe set. All the images are normalized and cropped into $200 \times 60$ pixels based on the manually labeled positions of the tip of the head (foremost point of an insect head in the dorsal view) and the end of the abdomen.

Table II lists the comparative rank-1 classification rates of CDP and LBP on 237 probe images against 60 gallery images. Without image partition, the histogram of the entire image (i.e., only one subregion size of $200 \times 60$ pixels) is used to represent an insect in this experiment. Thirty-two equally quantized histogram bins are used with the parameter of $P=8$ and $R=1$. It can be seen from the table that both the secondorder CDP and the third-order CDP perform better than the first-order LBP, indicating that the high-order CDP indeed extracts more discriminative information than the first-order LBP. These experimental results are consistent to our previous face recognition results, and further prove the effectiveness of the proposed method.

\section{CONCLUSIONS AND DISCUSSIONS}

This paper focuses on the micropattern based image representation and recognition initiated by Local Binary Pattern (LBP). We reveal that the LBP operator actually extracts the first-order derivatives of spatial images along the radial directions. We propose a new Circular Derivative Pattern (CDP) which can extract high-order derivative information of images along the circular directions. Through comparative experiments on face recognition and insect classification, we prove that the high-order CDP representation contains more detailed and more discriminative information than the first-order LBP representation for image recognition.

\section{ACKNOWLEDGMENT}

We would like to thank Dr Gary Kong from CRC for National Plant Biosecurity for his helpful discussions and insect imaging.

\section{REFERENCES}

[1] T. Ojala, M. Pietikäinen, and D. Harwood, "A Comparative Study of Texture Measures with Classification Based on Feature Distributions," Pattern Recognition, vol. 29, pp. 5159, 1996.

[2] T. Ojala, M. Pietikäinen, and T. Mäenpää, "Multiresolution Gray-Scale and Rotation Invariant Texture Classification with Local Binary Patterns," TPAMI, vol. 24, pp. 971-987, 2002.

[3] A. Hadid, M. Pietikäinen, and T. Ahonen, "A Discriminative Feature Space for Detecting and Recognizing Faces," CVPR, pp. 797-804, 2004.

[4] T. Ahonen, A. Hadid, and M. Pietikäinen, "Face Description with Local Binary Patterns: Application to Face Recognition," TPAMI, vol. 28, pp. 2037-2041, 2006.

[5] G. Zhao and M. Pietikäinen, "Dynamic Texture Recognition Using Local Binary Patterns with an Application to Facial Expressions," TPAMI, vol. 29, pp. 915-928, 2007.

[6] M. Heikkilä and M. Pietikäinen, "A Texture-Based Method for Modeling the Background and Detecting Moving Objects," TPAMI, vol. 28, pp. 657-662, 2006.

[7] S. Zhao, Y. Gao, and B. Zhang, "Sobel-LBP," ICIP, pp. 2144 2147, 2008.

[8] B. Zhang, Y. Gao, S. Zhao, and J. Liu, "Local Derivative Pattern Versus Local Binary Pattern: Face Recognition with High-Order Local Pattern Descriptor," TIP, vol. 19, pp. 533544, 2010.

[9] P.J. Phillips, H. Moon, S.A. Rizvi, and P.J. Rauss, "The FERET Evaluation Methodology for Face-Recognition Algorithms," TPAMI, vol. 22, pp. 1090-1104, 2000 